\title{
Gênero como marcador das relações de cuidado informal em saúde mental
}

\author{
Gender as a marker of informal care relations in \\ mental health
}

\author{
Luciane Prado Kantorski ${ }^{1}$ (D), Vanda Maria da Rosa Jardim ${ }^{1}$ (D), \\ Carlos Alberto dos Santos Treichel ${ }^{1}$ (D), Ana Paula Muller de Andrade ${ }^{1}$ (D), \\ Marta Solange Streicher Janelli da Silva ${ }^{1}$ (D), Valéria Cristina Christello Coimbra ${ }^{1}$ (D)
}

\begin{abstract}
Resumo
Introdução: A interface do gênero enquanto importante categoria no âmbito da saúde mental ainda é incipiente, ocasionando uma reificação de práticas que reproduzem desigualdades e assimetrias entre homens e mulheres cuidadores/as no contexto da atenção em saúde mental no Brasil. Objetivo: Discutir como o gênero tem marcado as práticas de cuidado desempenhadas pelas famílias, por meio da identificação de diferenças quanto ao perfil sociodemográfico, ao desenvolvimento das atividades de cuidado e às suas repercussões na vida dos familiares de acordo com o sexo dos indivíduos. Método: Estudo transversal conduzido com 1.242 familiares de usuários/as de Centros de Atenção Psicossocial. Verificou-se a prevalência de cada estrato das variáveis de acordo com o sexo dos indivíduos estudados utilizando o teste qui-quadrado para heterogeneidade. Resultados: Aspectos, como ausência da divisão da atividade de cuidado, sentimento de sobrecarga, avaliação ruim da qualidade de vida, insatisfação com as relações familiares e manifestação de transtornos psiquiátricos menores, foram mais prevalentemente encontrados entre as mulheres. Conclusão: Há diferenças importantes entre homens e mulheres cuidadores/as em saúde mental, em especial no que diz respeito às repercussões do cuidado na vida dessas pessoas.
\end{abstract}

Palavras-chave: gênero e saúde; cuidadores; saúde mental; serviços comunitários de saúde mental.

\begin{abstract}
Background: The gender interface as an important category in the field of mental health is still incipient. This results in the reification of practices that reproduce inequalities and asymmetries between men and women caregivers in the context of psychiatric care in Brazil. Objective: To discuss how gender has marked care practices performed by families through the identification of differences in socio-demographic profile, development of care activities and their repercussions on the family's life according to the sex of the individuals. Method: A cross-sectional study with 1242 relatives of users of Psychosocial Care Centers. We verified the prevalence of each variable stratum according to the sex of the individuals studied using chi-square test for heterogeneity. Results: Aspects such as absence of the division of care activity, feeling of overload, poor quality of life, dissatisfaction with family relationships, and manifestation of minor psychiatric disorders were more prevalent among the women accessed. Conclusion: There are important differences between men and women caregivers in mental health, especially regarding the repercussions of care on the lives of these people.
\end{abstract}

Keywords: gender and health; caregivers; mental health; community mental health services.

'Departamento de Enfermagem, Universidade Federal de Pelotas (UFPel) - Pelotas (RS), Brasil.

Trabalho realizado em 40 Centros de Atenção Psicossocial localizados na Região Sul do Brasil - Pelotas (RS), Brasil.

Endereço para correspondência: Luciane Prado Kantorski - Universidade Federal de Pelotas (UFPel), Rua Gomes Carneiro, 1 - Porto - CEP: $96010-610$ - Pelotas (RS), Brasil - Email: carlos-treichel@hotmail.com

Fonte de financiamento: nenhuma.

Conflito de interesses: nada a declarar.

Este é um artigo publicado em acesso aberto (Open Access) sob a licença Creative Commons Attribution, que permite uso, distribuição e reprodução em qualquer meio, sem restrições desde que o trabalho original seja corretamente citado. 


\section{INTRODUÇÃO}

A partir da reforma psiquiátrica, iniciada no país na década de 1980, ocorreram diversas mudanças nos processos de cuidado com as pessoas com transtornos mentais no Brasil. Entre essas mudanças, destaca-se o surgimento de serviços extra-hospitalares, como o Centro de Atenção Psicossocial (CAPS), que consiste em um serviço comunitário aberto do Sistema Único de Saúde regulamentado pelas Portarias GM/MS n $4.279 / 10$ e n 3.088/11, republicadas em 2013. O CAPS se constitui em um ordenador da rede de serviços em saúde mental e é responsável por acolher os indivíduos em sofrimento psíquico e suas famílias, oferecendo cuidados clínicos e de reabilitação psicossocial ${ }^{1,2}$.

Nesse contexto, houve uma significativa reconfiguração da participação da família no cuidado com as pessoas acometidas por algum tipo de sofrimento psíquico. Além de participarem como atores sociais importantes do processo de consolidação dos serviços substitutivos ao hospital psiquiátrico, os familiares foram convocados a compartilhar o cuidado com os profissionais e os próprios sujeitos ${ }^{1,2}$.

A família, na perspectiva psicossocial, é entendida como provedora de cuidado e demandante de cuidados, uma vez que integra a rede social e compõe o contexto sociocultural em que os sujeitos acometidos por algum transtorno mental estão inseridos. Assim, os familiares produzem saúde em conjunto com os demais atores sociais envolvidos nas práticas de cuidado em liberdade ${ }^{3}$.

Trabalhar com os familiares nesse contexto demanda esforços, já que as famílias que procuram os serviços que compõem a rede de atenção psicossocial apresentam diferentes configurações. Nesse sentido, marcadores sociais, como a classe, a etnia e, sobretudo, o gênero, marcam, de forma significativa, as práticas de cuidado ${ }^{4}$.

Quanto ao gênero, as mulheres são reconhecidas como maioria no que se refere à prestação de cuidados, por exemplo, mães, irmãs, cônjuges, avós, entre outras parentalidades, o que reflete determinada configuração de gênero que associa as atividades delas ao âmbito privado/doméstico, no qual o cuidado é demandado, à revelia das inúmeras transformações e conquistas das mulheres no âmbito público. Outrossim, tais mulheres acumulam tarefas públicas e privadas, em duplas e triplas jornadas de trabalho ${ }^{5}$.

O gênero, tal como propõe $\mathrm{Scott}^{6}$, não deve ser pensado apenas para dar sentido às diferenças percebidas entre homens e mulheres, mas para ir além e ser tomado como uma categoria que permita compreender os significados culturais e os sentidos atribuídos para homens e mulheres em suas relações. Nesse sentido, segundo a autora, o gênero seria um primeiro modo de dar significado às relações de poder. Tal abordagem ultrapassa os limites de uma interpretação relacionada estritamente ao sexo, ou seja, àquilo que estaria relacionado aos caracteres genitais de homens e mulheres, e propõe uma análise que contemple o caráter relacional entre ambos ${ }^{6}$.

A interface do gênero enquanto importante categoria no âmbito da saúde mental ainda é incipiente, como apontam Zanello e Andrade ${ }^{7}$; decorre daí uma reificação de práticas que reproduzem desigualdades e assimetrias entre homens e mulheres cuidadores/as no contexto da atenção em saúde mental no Brasil. Tal como apontam Gutierrez e Minayo ${ }^{3}$, quando essa discussão aparece, não tem o devido aprofundamento.

Diante da complexidade do desenvolvimento de um cuidado singular no âmbito da atenção em saúde mental, o objetivo deste artigo é discutir como o gênero tem marcado as práticas de cuidado desempenhadas pelas famílias, por meio da identificação de diferenças quanto ao perfil sociodemográfico, ao desenvolvimento das atividades de cuidado e às suas repercussões na vida dos familiares de acordo com o sexo dos indivíduos.

\section{MÉTODO}

Trata-se de um estudo transversal, realizado com familiares de usuários/as de Centros de Atenção Psicossocial (CAPS) do tipo I, II e III, parte integrativa de uma pesquisa de avaliação de serviços de saúde mental de base comunitária da região Sul do Brasil (CAPSUL II), realizada em 2011.

Os/as participantes deste estudo foram submetidos à aplicação de um formulário pré-estruturado composto por perguntas acerca de dados sociodemográficos, utilização dos serviços de saúde, condições de saúde, participação em atividades, características do cuidado prestado, sentimento de sobrecarga e avaliação de sua qualidade de vida.

A coleta de dados ocorreu em 40 CAPS do tipo I, II e III, distribuídos nos Estados do Sul do Brasil (Paraná, Santa Catarina e Rio Grande do Sul).

Para seleção dos serviços incluídos no estudo, respeitou-se a proporcionalidade de serviços em cada Estado, de forma que foram incluídos na amostra 12 serviços no Paraná, 10 em Santa Catarina e 18 no Rio Grande do Sul.

Com base no cálculo de amostra, pretendeu-se aplicar o questionário a 1.600 usuários/as de Centros de Atenção Psicossocial. Para prevalência, o cálculo de amostra considerou uma frequência estimada de $50 \%$, com margem de 3 pontos e alfa ( $\alpha$ ) de $5 \%$, resultando na necessidade de um $\mathrm{N}=1.066$. Já para associação, utilizando um poder de amostra de $90 \%$, com confiança de $95 \%$, relação de não expostos/expostos de $2: 1$, risco relativo de 1,3 e considerando prevalência de $40 \%$ em não exposto, obteve-se um indicativo de amostra de $\mathrm{N}=1.038$. Logo, acresceu-se ao maior $\mathrm{N}$ indicado $(\mathrm{N}=1.066) 50 \%$ de indivíduos a fim de considerar perdas e controle de fator de confusão.

A seleção dos entrevistados foi realizada por meio de amostragem não probabilística. Todos os familiares que 
frequentaram o serviço no período de coleta (uma semana) foram abordados. Além deles, a fim de completar a amostra, por meio dos registros dos serviços, foram rastreados familiares aos quais foram realizadas visitas domiciliares. O percentual de perdas no universo dos familiares correspondeu a $23 \%$, relacionado a recusas e dificuldades em encontrá-los; sendo assim, a população final acessada por este estudo foi de 1.242 familiares.

A coleta foi feita por 40 entrevistadores independentes, selecionados e treinados previamente. O controle de qualidade dos dados foi realizado na codificação dos instrumentos de coleta, na revisão efetuada pelos supervisores ao receber os questionários e na replicação de $5 \%$ das entrevistas realizadas. A entrada dos dados no banco ocorreu por meio de dupla digitação no software EPI-INFO 6.04, e as diferenças entre os dados foram comparadas, avaliadas e corrigidas, quando necessário.

Como desfecho, este estudo buscou identificar o perfil dos/as cuidadores/as e as repercussões do cuidado em suas vidas de acordo com a perspectiva de gênero. Foram incluídas neste estudo as variáreis: sexo (feminino e masculino), idade ( 18 a 25 anos, 26 a 40 anos, 41 a 50 anos e 51 anos ou mais), estado civil (com companheiro e solteiro), vínculo com o usuário (pais, irmãos, cônjuges, filhos e outros), escolaridade (sem escolaridade, até 4 anos de estudo, entre 5 e 8 anos de estudo, entre 9 e 11 anos de estudo e 12 anos de estudo ou mais), renda (até 1 salário mínimo, entre 1 e 2 salários mínimos, entre 2 e 3 salários mínimos e 3 salários mínimos ou mais), trabalho remunerado (possui trabalho remunerado e não possui trabalho remunerado), problemas de saúde (não possui e possui), divisão das atividades do cuidado (divide o cuidado e cuida sozinho), sentimento de sobrecarga (não sobrecarregado, pouco sobrecarregado, mais ou menos sobrecarregado e muito sobrecarregado), avaliação da qualidade de vida (boa e ruim), relação com a família (satisfeito, mais ou menos satisfeito e insatisfeito) e transtornos psiquiátricos menores (rastreio negativo e rastreio positivo). Para rastreamento de transtornos psiquiátricos menores, utilizou-se a escala Self-Reporting Questionnaire (SRQ20), tendo como base a validação brasileira da escala ${ }^{8}$, que adotou como ponto de corte $6 / 8$.

As análises foram conduzidas com o pacote estatístico Stata 11 (Stata Corp., College Station, Estados Unidos). Foi verificada a prevalência de cada estrato das variáveis de acordo com o sexo dos indivíduos estudados, utilizando-se o teste qui-quadrado para heterogeneidade a fim de identificar diferenças estatisticamente significativas entre os grupos $(\mathrm{p}<0,05)$.

O estudo foi submetido, sob ofício n ${ }^{\circ}$ 176/2011, pelo Comitê de Ética em Pesquisa da Faculdade de Enfermagem da Universidade Federal de Pelotas e aprovado por ele, seguindo as normas e as diretrizes regulamentadoras da pesquisa envolvendo seres humanos - Resolução CNS nº 196/96. Os aspectos deste estudo atendem também à Resolução CNS n 466/2012. Os princípios éticos foram assegurados por meio de: consentimento livre e esclarecido, garantia do direito de não participação na pesquisa e anonimato. Não houve conflitos de interesse na realização deste estudo.

\section{RESULTADOS}

A amostra do estudo compreendeu 842 mulheres (67,8\%) e 400 homens (32,2\%). Escolaridade foi a única característica estudada em que não houve diferença estatística entre os sexos; nas demais variáveis, todas as comparações por meio do teste qui-quadrado apresentaram $p$-valor $<0,05$. A distribuição entre sexos para todas as variáveis estudadas pode ser observada na Tabela 1.

Entre homens e mulheres, a faixa etária mais prevalente foi aquela cujos indivíduos possuíam de 41 a 50 anos (40,3\% e $49,3 \%$, respectivamente). No entanto, houve uma prevalência significativamente maior de indivíduos com 61 anos de idade ou mais do sexo masculino (36\%) em comparação ao sexo feminino $(24,2 \%)$.

A maior prevalência de indivíduos que possuíam companheiro entre homens foi de $73,7 \%$, enquanto entre as mulheres foi de $54,8 \%(\mathrm{p}<0,001)$.

Quanto ao vínculo com o indivíduo do qual são cuidadores/as, foi evidenciado que, entre as mulheres, a maior parte $(39,5 \%)$ era mãe. Já entre os homens, $22,2 \%$ referiram ser pai do indivíduo de que cuidavam, porém o vínculo mais prevalente foi o de cônjuge (44,5\%). Houve ainda uma diferença significativa entre os sexos quanto àqueles que referiram ser irmãos do indivíduo cuidado: entre os homens, foi de $9,5 \%$, enquanto, entre as mulheres, foi de $19,2 \%$.

Em relação à renda, os resultados do estudo apontam para diferenças estatísticas $(p=0,000)$ entre a renda familiar de cuidadores/as do sexo masculino e do sexo feminino. Embora a distribuição de ambos os sexos se concentrem majoritariamente entre os estratos de menor renda, há maior presença de mulheres nos dois estratos de menor renda.

Para ambos os sexos, houve maior prevalência de indivíduos sem trabalho remunerado em relação àqueles que possuíam algum vínculo trabalhista. No entanto, as questões de trabalho também estiveram marcadas por diferenças entre os sexos: $46,5 \%$ dos homens possuíam trabalho, enquanto $36 \%$ das mulheres afirmaram trabalhar, havendo diferença estatística significante $(\mathrm{p}<0,001)$.

Problemas de saúde foram mais prevalentes em mulheres, com $58 \%$, enquanto, nos homens, essa prevalência foi de $44,8 \%$ $(\mathrm{p}<0,001)$.

Em ambos os sexos, a maior parte dos entrevistados referiu ser o único cuidador do usuário do CAPS. No entanto, houve diferença estatística significante quanto à divisão do cuidado 
entre os sexos $(\mathrm{p}=0,001): 58,7 \%$ dos homens afirmaram ser o único cuidador, ao passo que, entre as mulheres, essa prevalência foi de $68,6 \%$.

Sentimento de sobrecarga foi evidenciado em maior intensidade entre as mulheres que compuseram a amostra, com 30,3\%, do que entre os homens, com 18,8\%. Já a maior prevalência de indivíduos não sobrecarregados foi maior entre os homens, com $54,1 \%$, do que entre as mulheres, com 43,5\%.

Quanto à avaliação da qualidade de vida, em ambos os sexos, a maior parte da amostra fez uma avaliação positiva. No entanto,

Tabela 1. Distribuição das variáveis analisadas de acordo com o sexo dos participantes dos Centros de Atenção Psicossocial da região Sul (CAPSUL) do Brasil, 2011

\begin{tabular}{|c|c|c|c|c|}
\hline & $\mathbf{N}$ & $\%$ em homens & $\%$ em mulheres & P-valor \\
\hline \multicolumn{5}{|l|}{ Idade } \\
\hline Até 25 anos & 101 & $9,0 \%$ & $7,7 \%$ & \multirow[t]{4}{*}{$<0,001$} \\
\hline 26 a 40 anos & 217 & $14,7 \%$ & $18,8 \%$ & \\
\hline 41 a 50 anos & 576 & $40,3 \%$ & $49,3 \%$ & \\
\hline 51 anos ou mais & 348 & $36,0 \%$ & $24,2 \%$ & \\
\hline \multicolumn{5}{|l|}{ Estado civil } \\
\hline Com companheiro & 755 & $73,7 \%$ & $54,8 \%$ & \multirow[t]{2}{*}{$<0,001$} \\
\hline Solteiro & 484 & $26,3 \%$ & $45,2 \%$ & \\
\hline \multicolumn{5}{|l|}{ Vínculo } \\
\hline Pais & 422 & $22,2 \%$ & $39,5 \%$ & \multirow[t]{5}{*}{$<0,001$} \\
\hline Irmãos & 200 & $9,5 \%$ & $19,2 \%$ & \\
\hline Cônjuges & 303 & $44,5 \%$ & $14,9 \%$ & \\
\hline Filhos & 190 & $16,0 \%$ & $15,0 \%$ & \\
\hline Outros & 127 & $7,8 \%$ & $11,4 \%$ & \\
\hline \multicolumn{5}{|l|}{ Escolaridade } \\
\hline Sem escolaridade & 133 & $9,6 \%$ & $11,3 \%$ & \multirow[t]{5}{*}{0,595} \\
\hline Até 4 anos de estudo & 551 & $47,2 \%$ & $43,5 \%$ & \\
\hline Entre 5 e 8 anos de estudo & 162 & $13,5 \%$ & $13,0 \%$ & \\
\hline Entre 9 e 11 anos de estudo & 276 & $20,3 \%$ & $23,4 \%$ & \\
\hline 12 anos de estudo ou mais & 111 & $9,4 \%$ & $8,8 \%$ & \\
\hline \multicolumn{5}{|l|}{ Renda } \\
\hline Até 1 salário mínimo & 200 & $11,3 \%$ & $20,4 \%$ & \multirow[t]{4}{*}{$<0,001$} \\
\hline Entre 1 e 2 salários mínimos & 390 & $32,3 \%$ & $35 \%$ & \\
\hline Entre 2 e 3 salários mínimos & 276 & $29,3 \%$ & $21,8 \%$ & \\
\hline 3 salários mínimos ou mais & 275 & $27 \%$ & $22,7 \%$ & \\
\hline \multicolumn{5}{|l|}{ Trabalho } \\
\hline Possui trabalho remunerado & 489 & $46,5 \%$ & $36,0 \%$ & \multirow[t]{2}{*}{$<0,001$} \\
\hline Não possui trabalho remunerado & 752 & $53,5 \%$ & $64,0 \%$ & \\
\hline \multicolumn{5}{|l|}{ Problema de saúde } \\
\hline Não possui & 575 & $55,2 \%$ & $42,0 \%$ & \multirow[t]{2}{*}{$<0,001$} \\
\hline Possui & 667 & $44,8 \%$ & $58,0 \%$ & \\
\hline \multicolumn{5}{|l|}{ Divisão das atividades do cuidado } \\
\hline Divide o cuidado & 429 & $41,3 \%$ & $34,4 \%$ & \multirow[t]{2}{*}{0,001} \\
\hline Cuida sozinho & 811 & $58,7 \%$ & $68,6 \%$ & \\
\hline \multicolumn{5}{|l|}{ Sentimento de sobrecarga } \\
\hline Não sobrecarregado & 581 & $54,1 \%$ & $43,5 \%$ & \multirow[t]{4}{*}{$<0,001$} \\
\hline Pouco sobrecarregado & 104 & $9,8 \%$ & $7,7 \%$ & \\
\hline Mais ou menos sobrecarregado & 224 & $17,3 \%$ & $18,5 \%$ & \\
\hline Muito sobrecarregado & 329 & $18,8 \%$ & $30,3 \%$ & \\
\hline \multicolumn{5}{|l|}{ Avaliação da qualidade de vida } \\
\hline Boa & 907 & $78,4 \%$ & $71,1 \%$ & \multirow[t]{2}{*}{0,007} \\
\hline Ruim & 328 & $21,6 \%$ & $28,9 \%$ & \\
\hline \multicolumn{5}{|l|}{ Relação com a família } \\
\hline Satisfeito & 981 & $84,0 \%$ & $76,8 \%$ & \multirow[t]{3}{*}{0,003} \\
\hline Mais ou menos satisfeito & 148 & $10,8 \%$ & $12,5 \%$ & \\
\hline Insatisfeito & 111 & $5,2 \%$ & $10,8 \%$ & \\
\hline Transtornos psiquiátricos menores & & & & \\
\hline Rastreio negativo & 384 & $62 \%$ & $49 \%$ & $<0,001$ \\
\hline Rastreio positivo & 780 & $38 \%$ & $51 \%$ & \\
\hline
\end{tabular}


$28,9 \%$ das mulheres avaliaram negativamente sua qualidade de vida, contra $21,6 \%$ dos homens ( $\mathrm{p}=0,007$ ).

No que se refere à satisfação com as relações familiares, embora, em ambos os sexos, os indivíduos satisfeitos tenham sido maioria, o estudo evidenciou maior nível de insatisfação do sexo feminino (10,8\%), em comparação com o sexo masculino (5,2\%).

O rastreio de transtornos psiquiátricos menores foi maior em mulheres (51\%) em relação aos homens (38\%). A diferença de prevalências entre os sexos apresentou significância estatística, evidenciando-se um p-valor $<0,001$.

\section{DISCUSSÃO}

Este estudo buscou identificar como o gênero tem marcado as práticas de cuidado desempenhadas pelas famílias no âmbito do cuidado informal em saúde mental, bem como discutir as diferenças apresentadas entre homens e mulheres nesse cenário.

Nessa perspectiva, cabe pontuar que a caracterização da população estudada pode ser entendida como ponto inicial para discussão, mostrando que, no âmbito dos familiares, a atenção em saúde mental tem uma marca importante de gênero. Assim como em outros estudos conduzidos com cuidadores familiares em saúde mental ${ }^{1,9,10}$, as mulheres corresponderam à maior parte da amostra.

O cuidado exercido majoritariamente pelas mulheres, na perspectiva de gênero, reflete valores morais e culturais que as interpelam cotidianamente. Associado a algo que seria da natureza das mulheres, o exercício dos cuidados vem sendo construído socialmente como tarefa delas ${ }^{11}$. Outro ponto que reforça essa perspectiva é a maior prevalência de mulheres como únicas cuidadoras de seus assistidos.

Esses dados vão ao encontro das discussões quanto à divisão sexual do trabalho, conforme apontado por Okin ${ }^{12}$. Para a autora, persiste uma ideia de divisão entre o trabalho público e o privado, sendo esse último fortemente desvalorizado e atribuído, principalmente, às mulheres.

Cabe destacar que uma limitação deste estudo é não contar com a informação do tempo de cuidado diário provido pelos cuidadores/as acessados/as. No entanto, estudos anteriores apontaram que, no âmbito da divisão do cuidado, as mulheres proviam cuidado diário em tempo aproximadamente duas vezes maior e cuidavam há um período quase três vezes maior que os homens ${ }^{13}$.

A perspectiva de que o gênero dos/as cuidadores/as permeia a definição das relações de cuidado dentro dos grupos familiares é tensionada pelas diferenças entre os sexos quanto ao tipo de parentesco com o indivíduo cuidado. Entre as mulheres acessadas neste estudo, boa parte eram mães, enquanto, entre os homens, a parcela mais expressiva foi de cônjuges.
Segundo Badinter ${ }^{14}$, sendo a maternagem uma construção social, histórica e não natural, pode ser exercida por homens e mulheres, ainda que estas assumam essa tarefa quase que exclusivamente nas sociedades ocidentais. A autora alega que apenas a maternidade, ou seja, $\mathrm{o}$ ato de parir, seria exclusivo das mulheres. Entretanto, não são apenas as mães que desempenham a tarefa do cuidado. Entre as/os irmãs/irmãos, tal como mostram os dados, as mulheres também prevaleceram.

A naturalização e a repetição dessa tarefa entre as mulheres decorrem do que Butler ${ }^{15}$ argumentou sobre o gênero como uma performance, ou seja, uma repetição estilizada de atos que acaba por ser reconhecida como natural e mesmo substancial.

É importante pontuar que essa naturalização se reflete também na idade com que ambos exercem o cuidado. As mulheres, ao assumirem o cuidado do familiar, ou o tratam como mais uma tarefa cotidiana, ou abrem mão do trabalho formal. Tal como mostram os dados, ainda que a faixa etária prevalente entre homens e mulheres tenha sido a mesma, a prevalência entre os homens com mais de 61 anos foi maior do que entre as mulheres.

Tal como argumenta $\operatorname{Okin}^{12}$ (p. 307) sobre a divisão trabalho por sexos:

Os homens são vistos como, sobretudo, ligados às ocupações da esfera da vida econômica e política e responsáveis por elas, enquanto as mulheres seriam responsáveis pelas ocupações da esfera privada da domesticidade e reprodução.

Corrobora, nesse sentido, o resultado que demonstra que a maior parte dos/das cuidadores/as não possuía trabalho remunerado. Contudo, quando o possuíam, a maior prevalência era entre os homens, sugerindo novamente a clássica divisão entre público/homens e privado/mulheres.

Quanto às repercussões do exercício do cuidado, é importante pontuar que este estudo encontrou maior prevalência de sobrecarga entre as mulheres, especialmente entre os indivíduos que se referiam muito sobrecarregados. Essa perspectiva vai ao encontro dos resultados prévios da literatura ${ }^{9}$ e reforça a ideia de que as perspectivas de gênero, em especial quanto à divisão do cuidado, têm impactado na vida dos/as cuidadores/as informais em saúde mental.

Entre as repercussões da atividade de cuidado, as mulheres apresentaram ainda de forma mais prevalente uma pior avaliação de sua qualidade de vida. Esse dado vai ao encontro dos achados de Larrañaga et al. ${ }^{16}$, que, em seu estudo, encontraram maiores chances de pior avaliação na qualidade de vida entre mulheres cuidadoras em saúde mental quando comparadas aos homens na mesma condição.

Destaca-se ainda a alta prevalência de transtornos psiquiátricos menores entre as mulheres estudadas, sendo esta significativamente superior àquela encontrada entre os homens. Essa perspectiva diz respeito a um importante processo de adoecimento dessas mulheres e vai ao encontro dos resultados de Santos ${ }^{17}$. A autora 
pontua que os múltiplos papéis desempenhados pela mulher na sociedade contribuem para um aumento significativo da incidência de transtornos mentais e comportamentais, uma vez que elas continuam com o fardo da responsabilidade que vem associado com os papéis de esposas, mães, educadoras e, por fim, cuidadoras.

Por se tratar de um estudo transversal, é importante levar em conta alguns aspectos, como causalidade reversa; contudo, em geral, a manifestação de transtornos psiquiátricos menores e de problemas de saúde em geral é sugerido como repercussão das atividades do cuidado ${ }^{18}$. Nesse sentido, esses aspectos configurariam repercussões com as quais os indivíduos do sexo feminino incluídos no estudo apresentaram maiores prevalências.

De forma geral, pode-se apontar que todos os resultados tiveram maior prevalência entre as mulheres. Portanto, aponta-se que a discussão das questões de gênero no âmbito do cuidado informal em saúde mental necessita ser amplamente divulgada, especialmente se levar em conta que, assim como argumentam Vilella et al. (2010) ${ }^{4}$, há muito tempo estudos têm apontado para a emergência de problemas de saúde entre as mulheres, decorrentes das desigualdades de gênero.

A limitada incorporação do gênero como um marcador social importante para a saúde tem impactado a saúde das mulheres, as quais têm suas questões geralmente associadas aos seus direitos reprodutivos e à noção de seu ciclo vital, definido pelas diversas fases de seu ciclo biológico, como gravidez, parto, pós-parto e menopausa ${ }^{19}$.

No campo do cuidado familiar em saúde mental, os estudos que consideram as questões relativas ao gênero como categoria de análise em geral estão relacionados ao cuidado de usuários de CAPS infantojuvenis, ou seja, crianças e adolescentes. Nesse sentido, destacam-se estudos de Bustamante e $\operatorname{Trad}^{20} \mathrm{e}$ Muylaert et al. ${ }^{21}$. Embora esses estudos trabalhem com uma população diferente da abordada neste artigo, existem pontos importantes de aproximação entre seus resultados, especialmente no que se refere à indicação de uma hegemonia da tradicional noção do feminino como lugar do cuidado.

Nesse sentido, cabe reforçar a necessidade de levar em conta as repercussões que a invisibilidade do gênero no âmbito da política pública de saúde mental tem na vida das mulheres, uma vez que elas, enquanto cuidadoras, demandam cuidados que, não raro, são construídos a partir de uma interpretação biologizante e psiquiatrizante ${ }^{22,23}$, devido à desconsideração das desigualdades e assimetrias de gênero presentes no contexto em que vivem.

Dessa forma, sugere-se que as práticas de cuidado compartilhadas por familiares com os serviços de saúde mental devam considerar o gênero como um marcador importante, já que, tal como apresentado, além de interferir significativamente na provisão dos cuidados dispensados pelas famílias a seus familiares, também apresenta impactos na demanda por cuidado por parte dos familiares.

Os resultados deste estudo apontam que há diferenças importantes entre homens e mulheres cuidadores/as em saúde mental, em especial no que diz respeito às repercussões do cuidado na vida dessas pessoas, tais como: sentimento de sobrecarga, avaliação ruim da qualidade de vida, insatisfação com as relações familiares e rastreio positivo para transtornos psiquiátricos menores - todos de forma mais prevalente entre as mulheres estudadas.

Considerando que parte dessas repercussões pode ter relação direta com a naturalização de construtos sociais relacionados ao gênero dos indivíduos, a limitada incorporação desse aspecto nas discussões em saúde, assim como demonstrado neste estudo, pode contribuir para um pior prognóstico de saúde física e mental, em especial das mulheres que assumem a função do cuidado.

Nesse sentido, sugere-se que os serviços de saúde mental adotem práticas que favoreçam cada vez mais a desconstrução da naturalização do papel de cuidador/a como atribuição preferencialmente feminina, por meio da inclusão de todos os membros da rede afetiva dos usuários nos projetos terapêuticos desenvolvidos.

Além desse aspecto, sugere-se ainda que a discussão de gênero seja levada em conta na abordagem das demandas de cuidadores/as, vivenciando repercussões do cuidado, a fim de que se levem em conta as assimetrias de gênero, rompendo, assim, com práticas biologizantes e psiquiatrizantes.

\section{REFERÊNCIAS}

1. Delgado PG. Sobrecarga do cuidado, solidariedade e estratégia de lida na experiência de familiares de Centros de Atenção Psicossocial. Physis. 2014;24(4):1103-26. http://dx.doi.org/10.1590/S0103-73312014000400007.

2. Bielemann VLM, Kantorski LP, Borges LR, Chiavagatti FG, Willrich JQ, Souza AS, et al. A inserção da família nos centros de atenção psicossocial sob a ótica de seus atores sociais. Texto Contexto Enferm. 2009;18(1):131-9. http://dx.doi.org/10.1590/S0104-07072009000100016.

3. Gutierrez D, Minayo MC. Produção de conhecimento sobre cuidados da saúde no âmbito da família, 2010. Cien Saude Colet. 2010;15(Supl 1):1497508. http://dx.doi.org/10.1590/S1413-81232010000700062.
4. Villela W, Monteiro S, Vargas E. A incorporação de novos temas e saberes nos estudos em saúde coletiva: o caso do uso da categoria gênero. Cien Saude Colet. 2009;14(4):997-1006. http://dx.doi.org/10.1590/S141381232009000400002 .

5. Abramo L. ¿Inserción laboral de las mujeres en américa latina: una fuerza de trabajo secundaria? Estud Fem. 2004;12(2):224-35. http://dx.doi. org/10.1590/S0104-026X2004000200013.

6. Scott J. Gênero: uma categoria útil para análise histórica. Educ Real. 1990;15(2):71-99. 
7. Zanello V, Andrade APM. Saúde mental e gênero: diálogos, práticas e interdisciplinaridade. Curitiba: Appris; 2014.

8. Mari JJ, Williams PA. Validity study of a psychiatric screening questionnaire (SRQ-20) in primary care in the city of Sao Paulo. Br J Psychiatry. 1986;148(1):23-6. http://dx.doi.org/10.1192/bjp.148.1.23. PMid:3955316.

9. Barroso SM, Bandeira M, Nascimento E. Sobrecarga de familiares de pacientes psiquiátricos atendidos na rede pública. Rev. Psiq. Clín. 2007;34(6):270-7.

10. Pegoraro RF, Caldana RHL. Mulheres, loucura e cuidado: a condição da mulher na provisão e demanda por cuidados em saúde mental. Saude Soc. 2008;17(2):82-94. http://dx.doi.org/10.1590/S0104-12902008000200009.

11. Marcondes WB, Rotenberg L, Portela LF, Moreno CRC. O peso do trabalho "leve" feminino à saúde. São Paulo Perspect. 2003;17(2):91-101. http:// dx.doi.org/10.1590/S0102-88392003000200010.

12. Okin SM. Gênero, o público e o privado. Estud Fem. 2008;16(2):305-32. http://dx.doi.org/10.1590/S0104-026X2008000200002.

13. Perrin PB, Panyavin I, Morlett Paredes A, Aguayo A, Macias MA, Rabago $\mathrm{B}$, et al. A disproportionate burden of care: gender differences in mental health, health-related quality of life, and social support in mexican multiple sclerosis caregivers. Behav Neurol. 2015;2015:283958. http://dx.doi. org/10.1155/2015/283958. PMid:26538818.

14. Badinter E. Um amor conquistado: o mito do amor materno. 2. ed. Rio de Janeiro: Nova Fronteira; 1985.

15. Butler J. Actos performativos y constitución del género: un ensayo sobre fenomenología y teoría feminista. In: Case SH, editor. Performing feminisms: feminist critical theory and theatre. Baltimore: Johns Hopkins; 1990. p. 296-314.

16. Larrañaga I, Martín U, Bacigalupe A, María Begiristáin J, José Valderrama M, Arregi B. Impacto del cuidado informal en la salud y la calidad de vida de las personas cuidadoras: análisis de las desigualdades de género. Gac Sanit. 2008;22(5):443-50. http://dx.doi.org/10.1157/13126925. PMid:19000525.

17. Santos AMCC. Articular saúde mental e relações de gênero: dar voz aos sujeitos silenciados. Cien Saude Colet. 2009;14(4):1177-82. http://dx.doi. org/10.1590/S1413-81232009000400023.

18. Treichel CAS, Jardim VMR, Kantorski LP, Vasem ML, Neutzling AS. Clustering of minor psychiatric disorders and burden among family caregivers of individuals with mental illness. Cien Saude Colet. 2016;21(2):585-90. http://dx.doi.org/10.1590/1413-81232015212.04392015.

19. MalufSW. Gênero, saúde e aflição: políticas públicas, ativismo e experiências sociais. In: Maluf SW, Tornquist CS, editores. Gênero, saúde e aflição: abordagens antropológicas. Florianópolis: Letras Contemporâneas; 2010.

20. Bustamante V, Trad LA. Participação paterna no cuidado de crianças pequenas: um estudo etnográfico com famílias de camadas populares. Cad Saude Publica. 2005;21(6):1865-74. http://dx.doi.org/10.1590/S0102311X2005000600036. PMid:16410873.

21. Muylaert CJ, Delfini PSS, Reis AOA. Relações de gênero entre familiares cuidadores de crianças e adolescentes de serviços de saúde mental. Physis. 2015;25(1):41-58. http://dx.doi.org/10.1590/S0103-73312015000100004.

22. Andrade APM. O gênero no movimento da reforma psiquiátrica brasileira. In: Maluf SW, Tornquist CS, editores. Gênero, saúde e aflição: abordagens antropológicas. Florianópolis: Letras Contemporâneas; 2010.

23. Andrade APM. Sujeitos e $(\mathrm{m})$ movimentos: uma análise crítica da reforma psiquiátrica brasileira na perspectiva dos experientes [tese]. Florianópolis: Universidade Federal de Santa Catarina; 2012.

Recebido em: Mar. 02, 2017 Aprovado em: Out. 28, 2018 